\title{
Ultrasound diagnosis of ulnar nerve entrapment by confirming baseline cross-sectional area measurement for normal and abnormal nerves
}

\author{
Authors: \\ Tania Roodt ${ }^{1}$ \\ Barbara van Dyk ${ }^{1}$ \\ Sylvia Jacobs ${ }^{1}$ \\ Affiliations: \\ ${ }^{1}$ Department of Radiography, \\ University of Johannesburg, \\ South Africa \\ Correspondence to: \\ Barbara van Dyk \\ Email: \\ bvandyk@uj.ac.za \\ Postal address: \\ PO Box 772, Roodepoort \\ 1725 , South Africa \\ Dates: \\ Received: 30 Oct. 2014 \\ Accepted: 15 May 2015 \\ Published: 05 Aug. 2015 \\ How to cite this article: \\ Roodt, T, Van Dyk, B, Jacobs, \\ S. Ultrasound diagnosis of \\ ulnar nerve entrapment \\ by confirming baseline \\ cross-sectional area \\ measurement for normal \\ and abnormal nerves. S \\ Afr J Rad. 2015;19(1); Art. \\ \#747, 7 pages. http://dx.doi. \\ org/10.4102/sajr.v19i1.747

\section{Copyright:} \\ C 2015. The Authors. \\ Licensee: AOSIS \\ OpenJournals. This work is \\ licensed under the Creative \\ Commons Attribution \\ License.
}

Read online:

Scan this QR

code with your

smart phone or

mobile device

to read online.
Background: Magnetic resonance imaging is most commonly employed, alongside electrodiagnostic studies, in the diagnosis of ulnar nerve entrapment. It is expensive, time consuming, not readily available to the general public, and limits imaging to a segment of the nerve at any given time. In contrast, high-frequency ultrasound is an inexpensive imaging modality with a flexible field of view through which the nerve can be traced. An ultrasound cross-sectional area (CSA) $>0.075 \mathrm{~cm}^{2}$ has previously been suggested as indicative of nerve entrapment.

Objectives: To confirm the suggested CSA measurement of $0.075 \mathrm{~cm}^{2}$ and discuss the difference in CSA measurement between abnormal nerves, nerves in the contra-lateral elbow of the same participant, and those of asymptomatic participants.

Methods: Ultrasonography was performed on both elbows of 25 patients with confirmed unilateral ulnar nerve entrapment and on 25 healthy controls for comparison. Three CSA measurements were taken of the ulnar nerve along its course, and the mean measurement was recorded.

Results: CSA measurements were significantly different between patients with ulnar nerve entrapment and healthy controls $(p<0.05)$. In our study, a CSA $>0.070 \mathrm{~cm}^{2}$ defined ulnar nerve entrapment at the elbow.

Conclusion: Ultrasound CSA measurement of the ulnar nerve is accurate in the diagnosis of ulnar nerve entrapment. The range of values and varied criteria previously reported call for standardisation of the procedure and CSA measurement. We suggest that a measurement of $0.070 \mathrm{~cm}^{2}$ be considered as a new baseline for the optimal diagnosis of ulnar nerve entrapment.

\section{Introduction and background}

High-frequency ultrasound is an inexpensive and non-invasive imaging modality that has become an appealing adjunct to electro-diagnostic studies in the evaluation of entrapment neuropathies. ${ }^{1,2}$ Currently, magnetic resonance imaging (MRI) is the most commonly employed imaging tool in the diagnosis of ulnar nerve entrapment; however, it is expensive, time consuming, not readily available to the general public and allows only a segment of the nerve to be imaged at any given time. ${ }^{3,4,5}$ In contrast, ultrasound provides a flexible field of view through which the nerve can be traced, and is able to clearly differentiate the nerve from the surrounding anatomical structures. ${ }^{1}$

Ulnar nerve entrapment may result in painful tingling or debilitating weakness of the hands or upper extremities. ${ }^{6}$ Peripheral nerves are vulnerable to compression at sites of fibrous and fibroosseous tunnels. The most common site for ulnar nerve entrapment is consequently where the nerve passes through the cubital tunnel owing to pathological narrowing of the tunnel. ${ }^{7}$

Recent technological advances in ultrasound have enabled investigation of the condition with high-resolution ultrasound along the path of the nerve with relative ease. . $3,8,9^{2}$ Chiou et al. suggested that an ulnar nerve cross-sectional area (CSA) measurement $>0.075 \mathrm{~cm}^{2}$ was indicative of nerve entrapment. ${ }^{2}$ This measurement was derived from a small sample of 24 patients of whom 14 were symptomatic and 10 asymptomatic, making the reliability of the suggested measurement of $0.075 \mathrm{~cm}^{2}$ questionable. ${ }^{2}$ Further research, on a larger sample, was thus indicated to test the reliability of the suggested CSA measurement.

\section{Objectives}

Our aims were to: (1) compare the previously suggested baseline CSA measurement of $0.075 \mathrm{~cm}^{2}$, for ulnar nerve entrapment, with findings from the present study to improve future diagnosis of 
the condition; (2) elaborate on the difference between normal and abnormal nerves by comparing the CSA measurement of abnormal ulnar nerves with the nerve in the contra-lateral elbow in the same participant and to that of asymptomatic participants; and (3) to report the effect of confounding factors on the size of the ulnar nerve.

\section{Method}

A prospective quantitative comparative research approach allowed statistical comparison of results in order to meet the objectives.

\section{Research population and sampling}

A quota non-probability sampling method was employed by recruiting patients with ulnar nerve entrapment, as confirmed by electro-diagnostic studies and referred for ultrasound examination of the elbow. Patients with a predisposition for ulnar nerve entrapment owing to a previous fracture, arthritis, elbow pain or surgery which complicates interpretation of the ultrasound image, were excluded from the study.

Asymptomatic participants who acted as controls were recruited from the general public.

\section{Research procedure}

Ultrasound scans were performed on a Toshiba Xario ultrasound unit (Model SSA-660A), using a $12 \mathrm{mHz}$ linear transducer with a small footprint. The ulnar nerve was examined both during flexion and extension of the elbow. During the acquisition of flexion views, the patient was seated next to the examination couch with the palm in contact with the examination table and the elbow rotated postero-laterally so that the humerus was at right angles to the trunk (Figures 1-2). This position accentuated the anatomical landmarks.

For extension views, the patient was positioned with the shoulder and elbow in extension. The dorsal aspect of the hand was in contact with the examination table and the palm inverted (Figure 3).

The examination commenced with a longitudinal survey of the ulnar nerve to search for oedematous thickening of the nerve or loss of normal ultrasound characteristics. Figure 4 demonstrates a normal ulnar nerve, at the level of the cubital tunnel, in a longitudinal plane with the elbow in flexion.

The probe was then rotated through $90^{\circ}$ into the transverse plane until the nerve appeared as an oval or round structure (Figure 5). To obtain this image, the medial aspect of the transducer (side of the marker on the transducer) was placed at the medial epicondyle and the lateral aspect of the transducer on the olecranon process (Figure 6).

Area measurements were performed on a transverse section of the nerve during flexion, by using the manual free-hand tracing method. Three successive CSA measurements

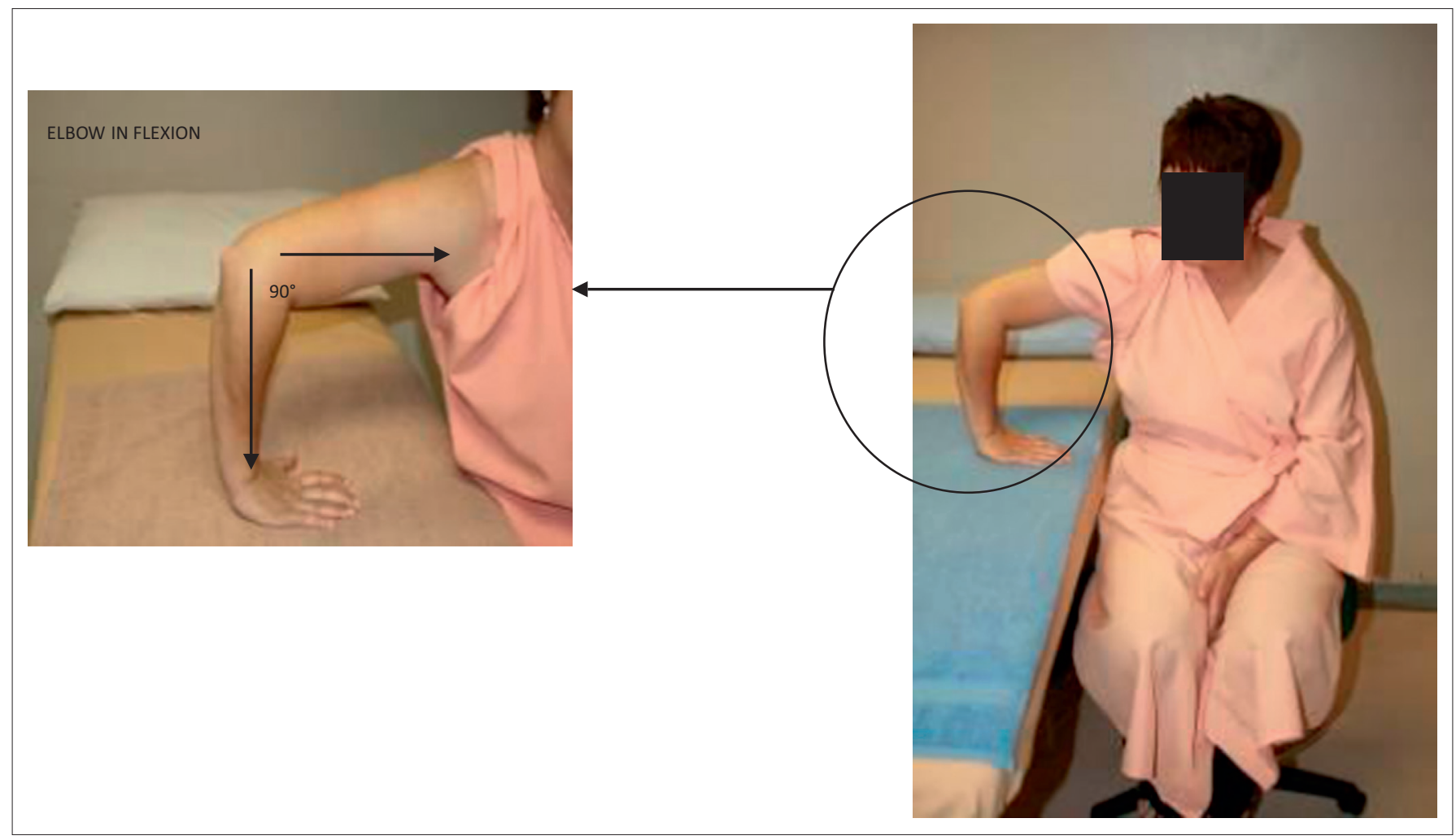




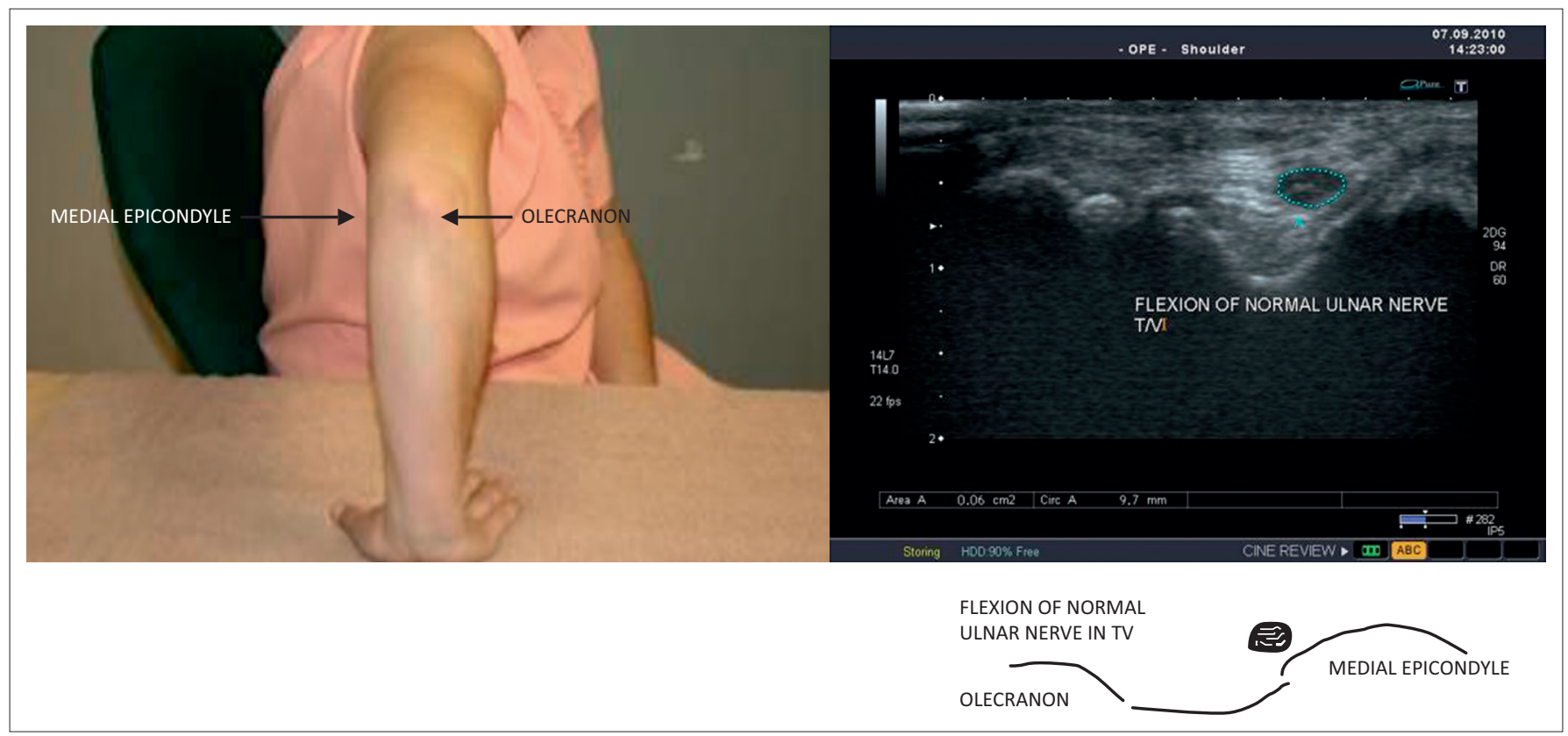

Source: T. Roodt.

FIGURE 2: Lateral projection of patient position for transverse and longitudinal imaging of the ulnar nerve with the elbow in $90^{\circ}$ flexion.

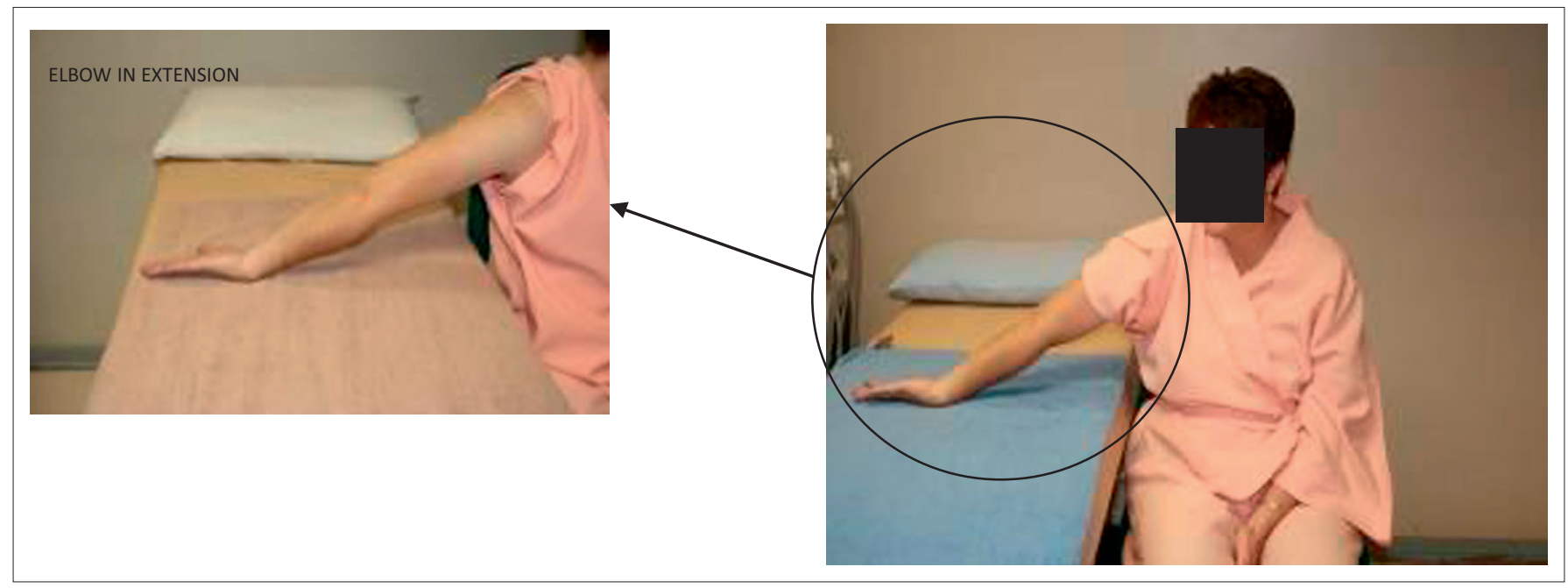

Source: T. Roodt.

FIGURE 3: Patient position for transverse and longitudinal imaging of the ulnar nerve with the elbow in full extension.

were obtained within the echogenic rim of the ulnar nerve (Figure 5) at:

1. the level of the medial epicondyle of the elbow

2. $50 \mathrm{~mm}$ proximal to the level of the medial epicondyle

3. $50 \mathrm{~mm}$ distal to the level of the medial epicondyle.

The same measurements were repeated with the elbow in extension, with the medial aspect of the transducer (side of the marker) placed at the medial epicondyle and the lateral aspect of the probe on the olecranon (Figure 7). This procedure was followed by three successive measurements of the contra-lateral arm in the same patient and compared with similar area measurements performed on control asymptomatic participants. Measurements were recorded on $\mathrm{CD}$ and the mean measurement was used for data analysis.

\section{Data collection and statistical analysis}

Twenty-five patients with unilateral ulnar nerve entrapment, confirmed by electro-diagnostic studies, were studied. Data were collected from the pathological nerve, contra-lateral arm of the same patient, and also from the dominant arm in 25 asymptomatic healthy participants for comparison.

Descriptive statistical analysis was performed with SPSS version 18.0, with ranges presented in graphical form. The Shapiro-Wilk test was used to examine the normality of data distribution in the small sample and to test the null hypothesis. Skewness of data necessitated the use of nonparametric tests to establish statistical significance. A $p$-value $<0.05$ was considered as statistically significant. 


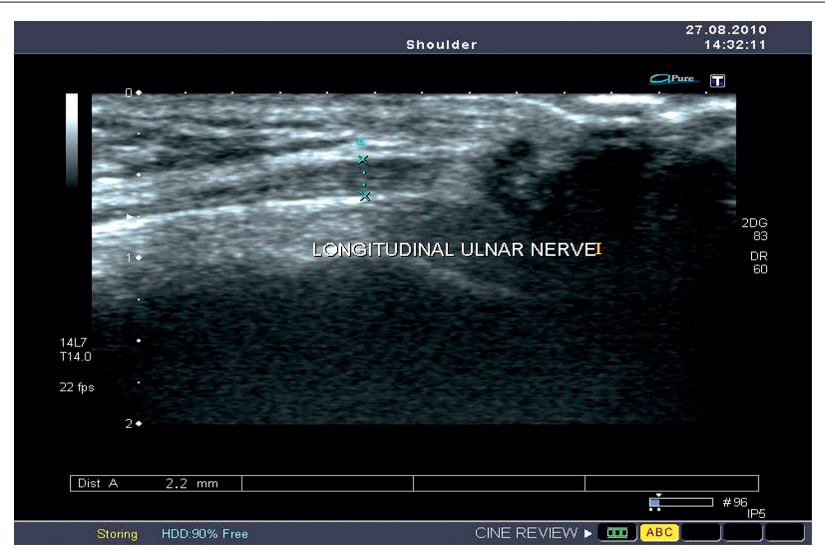

Source: T. Roodt.

FIGURE 4: Ultrasound image of the normal ulnar nerve in a longitudinal plane at the level of the medial epicondyle with the elbow in flexion.

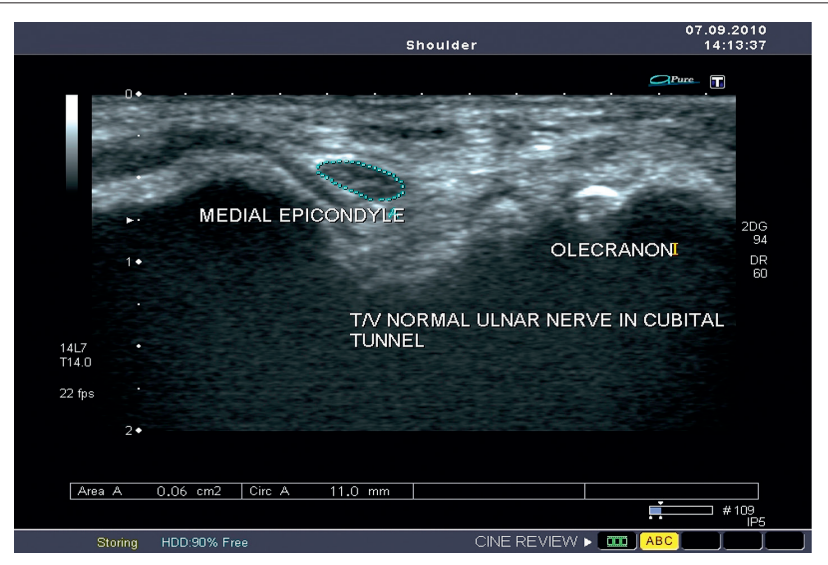

Source: T. Roodt.

FIGURE 5: Ultrasound image of the normal ulnar nerve (CSA $0.06 \mathrm{~cm}^{2}$ ) in a transverse plane, at the level of the medial epicondyle, with the elbow in flexion. The cross-sectional area was manually traced within the echogenic rim.

\section{Ethical considerations}

The study was approved by the Higher Degrees and Ethics Committees, Faculty of Health Sciences, University of Johannesburg. Participants were briefed on the purpose of the research and the procedure for data collection. Agreement to participate was indicated by signing informed consent while anonymity was ensured by using a research number.

\section{Validity and reliability}

All ultrasound scans were performed by the researcher, and measurements were confirmed by a radiologist to minimise data collector bias. Standardisation was ensured by the use of the same ultrasound unit with an automatic calibration function, a $12 \mathrm{mHz}$ linear transducer with a small footprint (PLT-1204AT), and a mechanical index of 0.6 accurate to $0.1 \mathrm{~mm}$.

\section{Results}

The ulnar nerve CSA measurement was significantly greater in symptomatic patients than in the asymptomatic

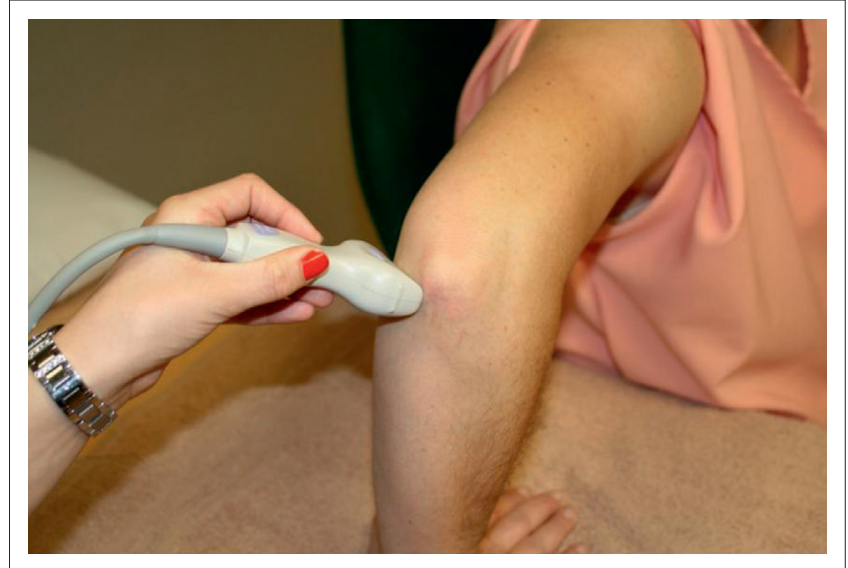

Source: T. Roodt.

FIGURE 6: Transducer position for a transverse section of the ulnar nerve at the level of the cubital tunnel with the elbow in flexion. The medial aspect of the transducer (side of the marker) was placed at the medial epicondyle and the lateral aspect of the probe on the olecranon.

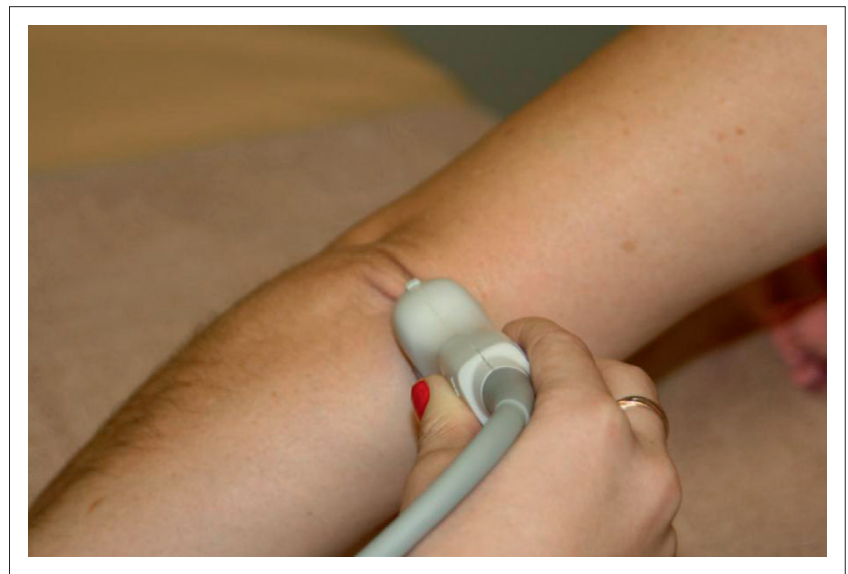

Source: T. Roodt.

FIGURE 7: Transducer position for a transverse section of the ulnar nerve at the level of the cubital tunnel with the elbow in extension. The medial aspect of the transducer (side of the marker) was placed at the medial epicondyle and the lateral aspect of the probe on the olecranon.

control group $(p<0.05)$ at all three measured levels. The bottom of the range in patients with confirmed ulnar nerve entrapment, with the elbow in flexion, was recorded as $0.07 \mathrm{~cm}^{2}$ (range $0.07 \mathrm{~cm}^{2}-0.25 \mathrm{~cm}^{2}$; median $0.10 \mathrm{~cm}^{2}$, mean $0.116 \mathrm{~cm}^{2}$ ) whilst the same measurement represented the maximum measurement for asymptomatic nerves in control participants (range $0.04 \mathrm{~cm}^{2}-0.07 \mathrm{~cm}^{2}$; median $0.051 \mathrm{~cm}^{2}$, mean $0.052 \mathrm{~cm}^{2}$ ). Measurements of the contra-lateral elbow overlapped between the measurements of the symptomatic and asymptomatic participants (range $0.05 \mathrm{~cm}^{2}-0.17 \mathrm{~cm}^{2}$; median $0.08 \mathrm{~cm}^{2}$, mean $0.09 \mathrm{~cm}^{2}$ ) (Figures 8-9; Tables 1-3).

CSA measurements in symptomatic and asymptomatic participants were significantly different $(p=0.001)$ at the level of the cubital tunnel. Measurements of the contra-lateral elbow overlapped between that of symptomatic and asymptomatic participants $(p=0.000)$.

A significant difference in CSA measurements $(p=0.000)$ was recorded between symptomatic and asymptomatic participants, $50 \mathrm{~mm}$ distal to the level of the cubital tunnel. 


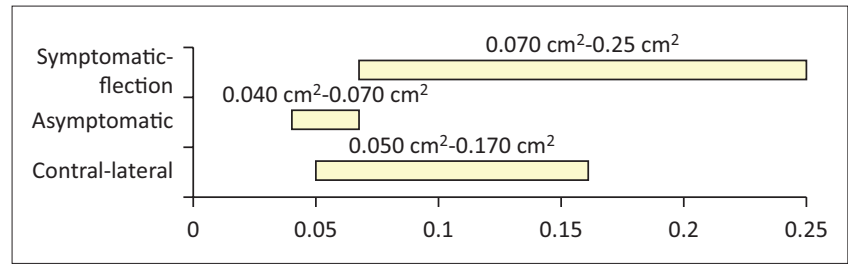

FIGURE 8: Ulnar nerve cross-sectional area measurement ranges in the cubital tunnel (flexion).

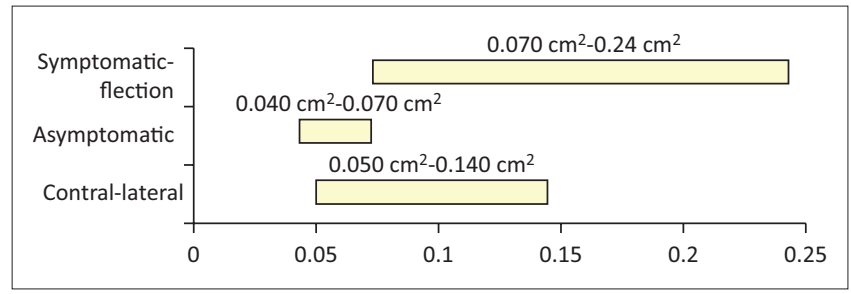

FIGURE 9: Ulnar nerve cross-sectional area measurement in the cubital tunne (extension).

TABLE 1a: Comparison of ulnar nerve measurements in symptomatic versus contra-lateral elbow and asymptomatic participants at the level of the cubital tunnel.

\begin{tabular}{|c|c|c|c|c|}
\hline Level & Diagnosis & Mean area measurement & $\pm S D$ & Range \\
\hline \multirow[t]{4}{*}{ Cubital tunnel - flexion } & Symptomatic elbow & $0.116 \mathrm{~cm}^{2}$ & $0.049 \mathrm{~cm}^{2}$ & $0.070-0.250 \mathrm{~cm}^{2}$ \\
\hline & Contra-lateral elbow & $0.091 \mathrm{~cm}^{2}$ & $0.032 \mathrm{~cm}^{2}$ & $0.050-0.170 \mathrm{~cm}^{2}$ \\
\hline & Asymptomatic elbow & $0.052 \mathrm{~cm}^{2}$ & $0.010 \mathrm{~cm}^{2}$ & $0.040-0.070 \mathrm{~cm}^{2}$ \\
\hline & Symptomatic elbow & $0.111 \mathrm{~cm}^{2}$ & $0.046 \mathrm{~cm}^{2}$ & $0.070-0.240 \mathrm{~cm}^{2}$ \\
\hline \multirow[t]{2}{*}{ Cubital tunnel - extension } & Contra-lateral elbow & $0.080 \mathrm{~cm}^{2}$ & $0.024 \mathrm{~cm}^{2}$ & $0.050-0.140 \mathrm{~cm}^{2}$ \\
\hline & Asymptomatic elbow & $0.054 \mathrm{~cm}^{2}$ & $0.010 \mathrm{~cm}^{2}$ & $0.040-0.070 \mathrm{~cm}^{2}$ \\
\hline
\end{tabular}

TABLE 1b: Comparison of ulnar nerve measurements in symptomatic versus contra-lateral elbow and asymptomatic participants at the level of the cubital tunnel.

\begin{tabular}{lcc}
\hline Test of normality & & \multicolumn{2}{c}{ Shapiro-Wilk } \\
\cline { 2 - 3 } & Statistic & Degrees of freedom \\
\hline Symptomatic: cubital tunnel - flexion & 0.81 & 25 \\
Asymptomatic: cubital tunnel - flexion & 0.833 & 0 \\
Symptomatic: cubital tunnel - extension & 0.76 & 25 \\
Asymptomatic: cubital tunnel - extension & 0.843 & 25 \\
\hline
\end{tabular}

TABLE 2a: Comparison of cross-sectional area measurements of the ulnar nerve, $50 \mathrm{~mm}$ distal to the level of the cubital tunnel.

\begin{tabular}{|c|c|c|c|c|}
\hline Level & Diagnosis & Mean area measurement & $\pm \mathrm{SD}$ & Range \\
\hline \multirow[t]{3}{*}{ Distal-flexion } & Symptomatic elbow & $0.080 \mathrm{~cm}^{2}$ & $0.030 \mathrm{~cm}^{2}$ & $0.040-0.180 \mathrm{~cm}^{2}$ \\
\hline & Contra-lateral elbow & $0.070 \mathrm{~cm}^{2}$ & $0.022 \mathrm{~cm}^{2}$ & $0.040-0.150 \mathrm{~cm}^{2}$ \\
\hline & Asymptomatic elbow & $0.049 \mathrm{~cm}^{2}$ & $0.010 \mathrm{~cm}^{2}$ & $0.030-0.070 \mathrm{~cm}^{2}$ \\
\hline \multirow[t]{3}{*}{ Distal - extension } & Symptomatic elbow & $0.080 \mathrm{~cm}^{2}$ & $0.027 \mathrm{~cm}^{2}$ & $0.040-0.190 \mathrm{~cm}^{2}$ \\
\hline & Contra-lateral elbow & $0.075 \mathrm{~cm}^{2}$ & $0.028 \mathrm{~cm}^{2}$ & $0.050-0.110 \mathrm{~cm}^{2}$ \\
\hline & Asymptomatic elbow & $0.052 \mathrm{~cm}^{2}$ & $0.009 \mathrm{~cm}^{2}$ & $0.040-0.070 \mathrm{~cm}^{2}$ \\
\hline
\end{tabular}

TABLE 2b: Comparison of cross-sectional area measurements of the ulnar nerve, $50 \mathrm{~mm}$ distal to the level of the cubital tunnel.

\begin{tabular}{lcc}
\hline Test of normality & & \multicolumn{2}{c}{ Shapiro-Wilk } \\
\cline { 2 - 3 } & Statistic & Degrees of freedom \\
\hline Symptomatic: distal - flexion & 0.845 & 25 \\
Asymptomatic: distal - flexion & 0.802 & 0.001 \\
Symptomatic: distal - extension & 0.875 & 25 \\
Asymptomatic: distal - extension & 0.721 & 25 \\
\hline
\end{tabular}

TABLE 3a: Comparison of cross-sectional area measurement of the ulnar nerve, $50 \mathrm{~mm}$ proximal to the level of the cubital tunnel.

\begin{tabular}{|c|c|c|c|c|}
\hline Level & Diagnosis & Mean area measurement & \pm SD & Range \\
\hline \multirow[t]{3}{*}{ Proximal - flexion } & Symptomatic elbow & $0.090 \mathrm{~cm}^{2}$ & $0.031 \mathrm{~cm}^{2}$ & $0.050-0.180 \mathrm{~cm}^{2}$ \\
\hline & Contra-lateral elbow & $0.075 \mathrm{~cm}^{2}$ & $0.028 \mathrm{~cm}^{2}$ & $0.040-0.150 \mathrm{~cm}^{2}$ \\
\hline & Asymptomatic elbow & $0.051 \mathrm{~cm}^{2}$ & $0.009 \mathrm{~cm}^{2}$ & $0.030-0.070 \mathrm{~cm}^{2}$ \\
\hline \multirow[t]{3}{*}{ Proximal-extension } & Symptomatic elbow & $0.091 \mathrm{~cm}^{2}$ & $0.033 \mathrm{~cm}^{2}$ & $0.060-0.190 \mathrm{~cm}^{2}$ \\
\hline & Contra-lateral elbow & $0.073 \mathrm{~cm}^{2}$ & $0.027 \mathrm{~cm}^{2}$ & $0.040-0.140 \mathrm{~cm}^{2}$ \\
\hline & Asymptomatic elbow & $0.053 \mathrm{~cm}^{2}$ & $0.008 \mathrm{~cm}^{2}$ & $0.040-0.070 \mathrm{~cm}^{2}$ \\
\hline
\end{tabular}

TABLE 3b: Comparison of cross-sectional area measurement of the ulnar nerve, $50 \mathrm{~mm}$ proximal to the level of the cubital tunnel.

\begin{tabular}{lcc}
\hline Tests of normality & \multicolumn{2}{c}{ Shapiro-Wilk } \\
\cline { 2 - 3 } & Statistic & Degrees of freedom \\
\hline Symptomatic: proximal - flexion & 0.878 & 25 \\
Asymptomatic: proximal - flexion & 0.861 & 0.006 \\
Symptomatic: proximal - extension & 0.848 & 25 \\
Asymptomatic: proximal - extension & 0.933 & 25 \\
\hline
\end{tabular}


TABLE 4: Comparison of mean cubital tunnel cross-sectional area measurements between flexion and extension in asymptomatic participants.

\begin{tabular}{|c|c|c|c|}
\hline Level & Mean area measurement & $\pm S D$ & Range \\
\hline Cubital tunnel - flexion & $0.052 \mathrm{~cm}^{2}$ & $0.010 \mathrm{~cm}^{2}$ & $0.040-0.070 \mathrm{~cm}^{2}$ \\
\hline Cubital tunnel - extension & $0.054 \mathrm{~cm}^{2}$ & $0.010 \mathrm{~cm}^{2}$ & $0.040-0.070 \mathrm{~cm}^{2}$ \\
\hline
\end{tabular}

The study likewise revealed a significant difference, although smaller, between CSA measurements $50 \mathrm{~mm}$ proximal to the level of the cubital tunnel with the arm in flexion $(p=0.003)$.

Confounding factors explored in the study included gender, race, height, age, weight, BMI, handedness, occupation and participation in sport. Owing to the small sample size, correlation coefficients, lying between -1 and +1 , were employed to determine the correlation between measurements and confounding factors. With a correlation coefficient $\geq 0.6$ indicating a strong correlation, a positive correlation was found between the CSA of the ulnar nerve and age (0.6), occupation (0.6) and weight/BMI (0.4) in both flexion and extension.

The unpaired $t$-test showed statistical significance $(p=0.004)$ between CSA measurements taken in flexion and extension in asymptomatic participants.

\section{Discussion}

Results of the present study concurred that the CSA of the ulnar nerve at all three levels was significantly increased in patients with confirmed ulnar nerve entrapment in comparison with asymptomatic participants. ${ }^{1,10}$ Although the majority of cubital tunnel CSA measurements in symptomatic patients were greater than the previously suggested 0.075 $\mathrm{cm}^{2}$, a measurement of $0.070 \mathrm{~cm}^{2}$ was recorded in two patients with confirmed ulnar nerve entrapment.

In keeping with results from previous studies, ${ }^{8,11,12}$ the CSA measurement of the contra-lateral elbow overlapped between the measurements recorded in symptomatic and asymptomatic participants for both flexion and extension of the elbow. This finding confirms the suspicion that ulnar nerve entrapment at the elbow occurs bilaterally in the majority of cases, although only one arm may present with symptoms of the condition. ${ }^{7,11,12}$ Populations at risk of flexion-induced ulnar neuropathy include office workers who habitually lean their flexed elbows against a desk or table. ${ }^{13}$

Moreover, in agreement with Thoirs, ${ }^{10}$ the CSA measurement of the ulnar nerve was greater when made with the elbow in extension than in flexion in the asymptomatic group (Table 4). This difference may be explained by the alteration in the length of the path of the nerve that occurs with elbow flexion, leading to changes in the shape and thickness of the nerve.

The results of our study concur with previous findings by showing that the ultrasound measurement of the ulnar nerve is susceptible to confounding factors arising from physical characteristics of the individual. ${ }^{2,9,10}$ However, owing to the small sample size, further investigation is needed to validate the magnitude of confounding factors on the ulnar nerve size.

\section{Limitations of the study}

Although the study population was more than double that of the previous study, 25 participants per group is still considered a small sample for meaningful statistical analysis, and thus remains a limiting factor in the present study. Furthermore, it was difficult to match the two groups, which led to participants in the symptomatic group being significantly older than the asymptomatic healthy group. Previous studies have suggested that ulnar nerve entrapment is a condition of middle age. ${ }^{10,14}$ The average age for the symptomatic group was 46 years (range 23-72) compared to an average of 36 years for the asymptomatic group (range 23-56).

\section{Conclusion and recommendations}

Whilst an electro-diagnostic study provides detailed physiological information, ultrasound provides detailed anatomical information of nerves. High-frequency ultrasound examination of the elbow is useful to detect soft tissue and bony abnormalities whilst providing information on adjacent anatomical structures. ${ }^{3}$

Ultrasonography is faster and less expensive than a comparable MRI test, is not associated with exposure to ionising radiation, and has no known adverse biological effects. ${ }^{3}$ Despite an increasing number of published articles, many practitioners still remain unaware of the effectiveness of high-resolution ultrasound for assessing peripheral nerves.

Although the numbers in our sample are too small to prove statistical significance, the results of the study suggest that a CSA measurement of $0.070 \mathrm{~cm}^{2}$ should be considered as a new baseline to differentiate between normal nerves and nerves affected by entrapment pathology, for the optimal diagnosis of ulnar nerve entrapment. With a new cut-off value of $0.070 \mathrm{~cm}^{2}$, it can be argued that patients with ulnar nerve entrapment might have been compromised if the previously suggested value of $0.075 \mathrm{~cm}^{2}$ had been adhered to. ${ }^{1}$ Further research on a bigger study population may be indicated to confirm our findings.

Furthermore, the enlargement of the asymptomatic contralateral nerve may indicate an underlying pathological condition or an habitual action, such as leaning on the elbow, that makes the nerve more vulnerable to injury.

The broad range of normative values reported in the literature $^{15}$ and varied criteria used in the diagnosis of 
ulnar nerve entrapment, call for standardisation of the ultrasound procedure and CSA measurement. The results of the present study demonstrate that the ulnar nerve can be reliably measured in cross-section when a closely controlled positioning and measurement protocol is followed.

In conclusion: the ultrasound CSA measurement of the ulnar nerve, made at the level of the medial epicondyle, is found to be a robust discriminator of ulnar nerve entrapment, making it a useful diagnostic test for the evaluation and diagnosis of ulnar nerve entrapment.

\section{Competing interests}

The authors declare that they have no financial or personal relationships which may have inappropriately influenced them in writing this article.

\section{Authors' contributions}

As the primary researcher of the Master's project from which the present article emanated, T.R. (University of Johannesburg) wrote the present article with thorough concern for the design, interpretation of data, and critical revision of the intellectual content to take public responsibility for it. She approves it for publication.

As supervisor of the research project, B.v.D. (University of Johannesburg) participated sufficiently in the conception and design of the present work, the data analysis (where applicable), critical review of the intellectual content and the writing of the article to take public responsibility for it. She reviewed the final version of the article and approves it for publication.

As co-supervisor of the Master's project, S.J. (University of Johannesburg) participated sufficiently in the conception, design, interpretation of data, critical revision of the intellectual content and the writing of the article to take public responsibility for it. She approves it for publication.

\section{References}

1. Ginn SD, Cartwright MS, Chloros GD, et al. Ultrasound in the diagnosis of a median neuropathy in the forearm: Case report. J Brachial Plex Peripher Nerve Inj. 2007;2:23.

2. Chiou H, Chou Y, Cheng S, et al. Cubital tunnel syndrome: Diagnosis by highresolution ultrasonography. J Ultrasound Med. 1998;17:643-648.

3. Introcasco JH, van Holsbeeck MT. Sonography of the elbow, wrist and hand. 2nd ed. Mosby, Maryland Heights: 2001; pp. 539-540, 528-529.

4. Maravilla KR, Bowen BC. Imaging of the peripheral nervous system: Evaluation of peripheral neuropathy and plexopathy. Am J Neuroradiol. 1998;19:10111023.

5. Steinback LS, Chung CB. MRI of the upper extremity. Philadelphia: Lippincott, Williams \& Wilkins; 2010; p. 192

6. Bayramoglu M. Entrapment neuropathies of the upper extremity. J Neuroanatomy. 2004;3:18-24.

7. Hochman M, Zilberfarb JL. Nerves in a pinch: Imaging of nerve compression syndromes. Radiol Clin North Am. 2004;42:221-245. http://dx.doi.org/10.1016/ S0033-8389(03)00162-3

8. Beekman R, Schoemaker MC, van der Plas JPL, et al. Diagnostic value of highresolution sonography in ulnar nerve neuropathy at the elbow. Neurology. 2004;62:767-773. http://dx.doi.org/10.1212/01.WNL.0000113733.62689.0D

9. Martinoli C, Bianchi S, Gandolfo N, Valle M, Simonetti S, Derchi LE. US of nerve entrapment in osteofibrous tunnels of the upper and lower limbs. Radiographics. 2000;20:S199-S217. http://dx.doi.org/10.1148/radiographics.20.suppl_1. g00oc08s199

10. Thoirs K, Williams MA, Phillips M. Systemic review of sonographic measurements of the ulnar nerve at the elbow. J Diagn Med Sonogr. 2007;23:255-262. http:// dx.doi.org/10.1177/8756479307306110

11. Pais $P$, Fodor D, Fisher T. Bilateral cubital tunnel syndrome: Case report with review of the literature. Med Ultrasonogr. 2009;11:31-36.

12. Dellon AL, Hament W, Gittelshon A. Non-operative management of cubital tunnel syndrome: An 8-year prospective study. Neurology. 1993;43:1673-1677. http:// dx.doi.org/10.1212/WNL.43.9.1673

13. Miller TT, Reinus WR. Nerve entrapment syndromes of the elbow, forearm, and wrist. Am J Roentgenol. 2010;195:585-594. http://dx.doi.org/10.2214/ AJR.10.4817

14. Yoon JS, Hong S, Kim B, et al. Ulnar nerve and cubital tunnel ultrasound in ulnar neuropathy at the elbow. Arch Phys Med Rehabil. 2008;89:887-889. http:// dx.doi.org/10.1016/j.apmr.2007.10.024

15. Koenig RW, Pedro MT, Heinen CPG, et al. High-resolution ultrasonography in evaluating peripheral nerve entrapment and trauma. Neurosurg Focus. 2009;26:1-6. http://dx.doi.org/10.3171/FOC.2009.26.2.E13 